\title{
Study of structural and optical properties of CdS:Mn quantum dots
}

\author{
Piyali Maity $^{1}$, Shiv Kumar ${ }^{1}$, Ranjan Kumar Singh ${ }^{1}$, Sandip Chatterjee ${ }^{2}$ \\ and Anup Kumar Ghosh ${ }^{1 *}$
}

${ }^{1}$ Department of Physics, Institute of Science, Banaras Hindu University, Varanasi-221005, India;

${ }^{2}$ Department of Applied Physics, Indian Institute of Technology (Banaras Hindu University), Varanasi-221005, India

*Email: akghosh@bhu.ac.in; anupkg66@gmail.com

A systematic study has been carried out to understand the influence of Manganese (Mn) doping on various properties of CdS QDs. Cd 1 ${ }_{\mathrm{x}} \mathrm{Mn}_{\mathrm{x}} \mathrm{S}(0 \leq \mathrm{x} \leq 0.06)$ QDs have been prepared at moderate low temperature by chemical precipitation method. X-ray diffraction (XRD) pattern, transmission electron microscopy (TEM) and Raman spectroscopy have been employed for phase identification and structural characterization of pure and Mn-doped CdS QDs. Fourier transform infrared (FTIR) spectroscopy, UV-Vis spectroscopy and photoluminescence (PL) spectroscopy have been used to study optical properties. Figure 1 shows Fluoroscence under UV light of pure CdS QDs.

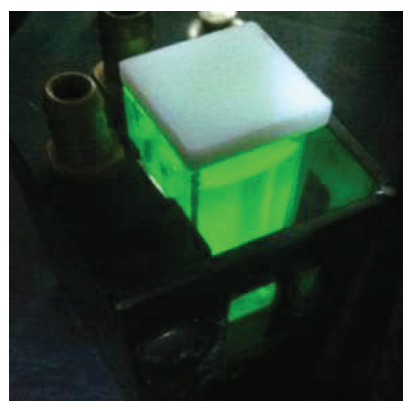

Figure 1: Fluoroscence under UV light of pure sample

X-ray diffraction reveals that both undoped and Mn-doped CdS QDs exhibit zinc-blende (JCPDS 75-0581) structure without any impurity phase. The size of the particle in the range of 2-4 $\mathrm{nm}$ has been confirmed by XRD and TEM measurements (Figure 1). Interplaner spacing and size of CdS QDs increase slightly with increasing Mn-concentration. TEM images demonstrated nearly monodisperse size distributions of as prepared CdS QDs. Raman spectra show two prominent peaks viz. 1-LO (stronger) and 2-LO (weaker) at frequencies $\sim 297$ and $\sim 597$ respectively
(Figure 2). The peak 1-LO decreases strongly with increasing Mn-concentration due to increased compositional and structural shortrange disorder. The absorption band observed at $\sim 620 \mathrm{~cm}^{-1}$ in FTIR spectra is assigned to $\mathrm{Cd}-$ $\mathrm{S}$ bond stretching. Band gap of the samples have been measured by UV-Vis spectroscopy which shows that the band gap is increasing linearly $(\sim 2.42 \mathrm{eV}$ for $\mathrm{x}=0$ to 6$)$ with Mnconcentration. The blue shift shows the quantum confinement effect, as well as $\mathrm{Mn}$ concentration effect.

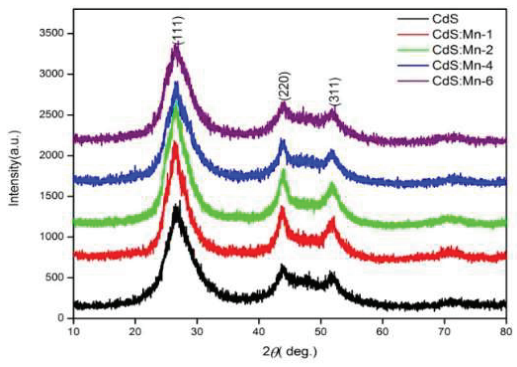

Figure 2: XRD pattern of $\mathrm{Cd}_{1-\mathrm{x}} \mathrm{Mn}_{\mathrm{x}} \mathrm{S}(0 \leq \mathrm{x} \leq 6)$

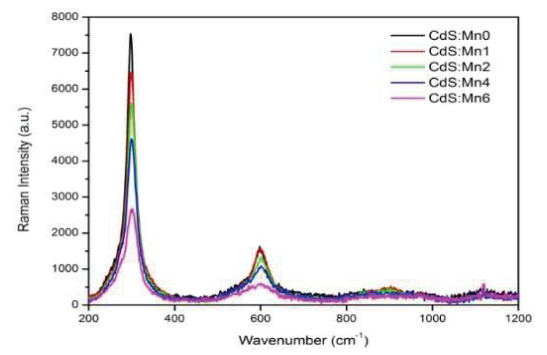

Figure 3: Raman spectra of $\mathrm{Cd}_{1-\mathrm{x}} \mathrm{Mn}_{\mathrm{x}} \mathrm{S}(0 \leq \mathrm{x} \leq 6)$

\section{References}

1. Xiao et al. J. Am. Chem. Soc. 136, 1559-1569 (2014)

2. Zeng et al. Chemistry of Materials 22, 2107(2010)

3. Hazarika et al. Physical Review Letters 110, 267401 (2013)

4. Silva et al. J. Phys. Chem. C 117, 1904 (2013) 